\title{
COMPARACIÓN MOLECULAR DE POBLACIONES DE CHILE (Capsicum spp.) DE TABASCO Y CHIAPAS, MÉXICO
}

\author{
Yasmín Araceli Gálvez M. ${ }^{1}$, María Esther Cea M. ${ }^{2}$, Julia María Lesher G. ${ }^{1}$, Luis Latournerie- \\ Moreno $^{3}$, Eusebio Martínez-Moreno ${ }^{4}$, José Luis Martínez S. ${ }^{1}$ y \\ Guillermo Castañón-Nájera ${ }^{1}$
}

\begin{abstract}
RESUMEN
Dada la importancia que tiene el chile en el sureste de la república mexicana, los objetivos de la investigación fueron caracterizar molecularmente, estimar el polimorfismo y la estructura genética de 21 poblaciones de Capsicum spp. de los estados de Tabasco y Chiapas. Se sembraron semillas de las poblaciones y a los 40 días después de la germinación, se cortaron dos hojas jóvenes por planta de 10 individuos de cada población. Las 20 hojas de cada población se mezclaron y de ellas se tomaron tres muestras de 0,5 g de tejido vegetal para extraer el ADN. Se usaron cuatro marcadores microsatélites (SSR) para estimar la diversidad genética. Se detectaron 229 alelos, de ellos 70 fueron polimórficos. El marcador Hpms1-106 detectó 38,8 \% de polimorfismo, y HpmsCaSIG-19 el menor polimorfismo (20,55 \%). El AMOVA explicó 13,0 \% de la variabilidad entre poblaciones, y los individuos dentro de las poblaciones el 87,0 \% restante. El estadístico $\mathrm{F}_{\mathrm{ST}}=0,176$ indicó que la diferenciación genética entre poblaciones es grande, el $\mathrm{F}_{\mathrm{IS}}=-0,448$ que las poblaciones poseen exceso de heterocigotos, y el $\mathrm{F}_{\mathrm{IT}}=-0,193$, que los individuos de cada población mostraron efecto moderado de apareamiento no aleatorio. El análisis cluster aglomeró a las poblaciones evaluadas en seis grupos. El cluster I agrupó 13 poblaciones, y dentro de éstas, Amashito Cerro Blanco y Colmillo de Lagarto El Porvenir mostraron ser las más parecidas genéticamente. En el cluster VI, Pico de Paloma Miahuatlán fue diferente al resto de las poblaciones. Los marcadores microsatélites fueron útiles para analizar la diversidad genética de las poblaciones de chile evaluadas.
\end{abstract}

Palabras clave adicionales: Análisis molecular, C. annuun, C. frutescens, diversidad genética, estructura de las poblaciones, marcadores moleculares

\begin{abstract}
Molecular comparison of chili populations (Capsicum spp.) from Tabasco and Chiapas, Mexico

Based on the importance of chile in the Southeast of Mexico, the objectives of this research were to characterize molecularly, and estimate the polymorphism and genetic structure of 21 populations of Capsicum spp. of Tabasco and Chiapas States, Mexico. After 40 days of germination of the seeds collected, two young leaves of each population were cut per each of 10 plants. The 20 leaves were mixed and three samples of $0.5 \mathrm{~g}$ were taken to extract the DNA. Four microsatellite markers (SSR) to determine the genetic diversity. A total of 229 alleles were detected, and 70 were polymorphics. The marker Hpms1-106 detected $38.8 \%$ polymorphism, and HpmsCaSIG-19 the lowest polymorphism (20.55\%). The AMOVA explained $13.0 \%$ of the variability among populations, and individuals within populations explained the remaining $87.0 \%$. The statistics F $_{\mathrm{ST}}=0.176$, showed a large differentiation between populations. The $\mathrm{F}_{\mathrm{IS}}=-0.448$ showed an excess of heterozygous in populations, and the $\mathrm{F}_{\mathrm{IT}}=-0.193$ showed that individuals in each population have moderate non-random mating effect between them. Cluster analysis grouped all evaluated chili populations into six clusters. The cluster I grouped 13 populations, some of these such as Amashito Cerro Blanco and Colmillo de Lagarto El Porvenir showed to be the most genetically similar. In the cluster VI, Pico de Paloma Miahuatlán showed to be different from the rest of populations. Microsatellite markers were useful to analyze the genetic diversity of these chili populations.
\end{abstract}

Additional keywords: C. annuun, C. frutescens, genetic diversity, molecular analysis, molecular markers, populations structure

Recibido: Junio 3, 2020

Aceptado: Noviembre 13, 2020

${ }^{1}$ División Académica de Ciencias Biológicas, Universidad Juárez Autónoma de Tabasco, Villahermosa, Tabasco, México.e-mail: yasminaraceli-gm@hotmail.com; lesher23@yahoo.com; jose.martinez@ujat.mx; guillermo_corazón_valiente@hotmail.com, guillermo.castanon@ujat.mx (autor de correspondencia)

${ }^{2}$ Universidad Agraria de la Habana Fructuoso Rodríguez Pérez. San José de Las Lajas, Mayabeque, Cuba. e-mail: mariacea@nauta.cu

${ }^{3}$ Instituto Tecnológico de México, Conkal, Yucatán, México. e-mail: sayilhahil@yahoo.com.mx.

${ }^{4}$ División Académica de Ciencias Agropecuarias, Universidad Juárez Autónoma de Tabasco.

e-mail: martinezmoreno9@gmail.com 


\section{INTRODUCCIÓN}

Entre las hortalizas de mayor consumo en el mundo se encuentra el género Capsicum, conocido comúnmente como chile, perteneciente a la familia de las Solanáceas (González et al., 2011). A Capsicum se le considera la hortaliza de mayor impacto económico y social. En México el chile tiene importancia cultural, social y económica, su distribución es amplia, su consumo per cápita es entre 8 y 9 kg (Castellón et al., 2012).

México es centro de domesticación de diversas especies vegetales, dentro de las que sobresale el género Capsicum, (Latournerie et al., 2002; Martínez et al., 2010). Al respecto MacNeish (1964), indica que el chile fue una de las primeras plantas domesticadas en América, que se usa como especia, condimento o verdura, ornamental, medicinal, como colorante alimenticio por su alto valor nutrimental en la dieta humana, y en la industria cosmetológica y farmacéutica (Chamikara et al., 2015; Xiao-min et al., 2016; Massot y Barbieri, 2016; Haralayya y Asha, 2017; Konishi et al., 2019).

Los métodos para analizar la diversidad genética han evolucionado de manera gradual, anteriormente los estudios se basaban en caracteres morfológicos, y en Capsicum la caracterización morfológica se ha realizado para identificar poblaciones silvestres, criollas (como Colmillo de lagarto, Huacle, Copi) o comerciales, también se han realizado evaluaciones electroforéticas de variantes bioquímicas y, más recientemente, análisis moleculares de las secuencias de ADN (Baltazar, 1997).

La caracterización morfológica ha sido cuestionada por el hecho de que algunos caracteres morfológicos son afectados por el ambiente, por considerarla una metodología ineficiente, costosa, y por requerir de más tiempo en su medición (Haralayya y Asha, 2017; De Vicente y Fulton, 2003). Asimismo, la caracterización morfológica en ocasiones no es capaz de detectar diferencias entre variedades con comportamiento agronómico diferente. Ejemplo de ello, es lo reportado por Kwon et al. (2007), quienes al estudiar descriptores morfológicos en variedades de chile, no encontraron diferencias entre ellas aunque eran agronómicamente distintas, dado que presentaban semejanza morfológica. Investigaciones en las que se estudió la divergencia genética entre marcadores morfológicos y moleculares en Capsicum spp., son la de Baba et al. (2015), en que se caracterizó chile habanero con datos morfológicos de fruto y moleculares con marcadores AFLP. Por su parte, Carvalho et al. (2017) estimaron la variabilidad genética de colecciones de germoplasma brasileño de Capsicum frutescens, con caracteres morfológicos y marcadores moleculares SSRs. Thul et al. (2012) realizaron el análisis de la diversidad genética de Capsicum spp. en características florales y con marcadores RAPDs e ISSR. Asimismo, López et al. (2019), con marcadores ISSR, detectaron alto nivel de polimorfismo y permitieron la diferenciación genética del complejo C. annuum. Mientras que Rahevar et al. (2019), reportaron que de los 26 marcadores usados en su estudio, sólo 13 de ellos identificaron polimorfismo en los cultivares de chile evaluados.

Por la importancia que tiene el chile en el sureste de la república mexicana, los objetivos de la investigación consistieron en evaluar la diversidad genética de poblaciones de $C$. annuum $\mathrm{L}$. y C. frutescens $\mathrm{L}$. mediante marcadores microsatélites (SSRs), estimar el polimorfismo, la estructura genética y la eficiencia de estos marcadores en chiles silvestres y criollos colectados en los estados de Tabasco y Chiapas.

\section{MATERIALES Y MÉTODOS}

Material vegetal. Se colectó 21 poblaciones de $C$. annuum L. y C. frutescens L., el nombre con el que los lugareños identifican a cada población y el sitio de colecta del material evaluado se muestran en el Cuadro 1. Cada una de las poblaciones germinó en charolas de poliestireno de 200 cavidades, previamente desinfectadas con cloro al $5 \%$. Se empleó turba como sustrato para la germinación y se depositó una semilla por cavidad. Después de la siembra, las charolas o bandejas se colocaron en el invernadero que se encuentra en la División Académica de Ciencias Biológicas, de la Universidad Juárez Autónoma de Tabasco, en Villahermosa, Tabasco, México.

Extracción de ADN y amplificación de microsatélites o secuencias simples repetidas. A los 40 días de edad de las plantas, se seleccionaron 10 plántulas de cada población, y se 
Gálvez et al. Comparación de poblaciones de Capsicum de Tabasco y Chiapas, México

les cortaron dos hojas jóvenes por planta, las 20 hojas se mezclaron y de ellas se tomó tres repeticiones de $0,5 \mathrm{~g}$ de tejido. Cada muestra se trituró con nitrógeno líquido en un mortero con pistilo de porcelana. Para la extracción del ADN se utilizó el kit comercial Wizard Genomic DNA Purification (Promega), y el método de Dellaporta et al. (1983).

Cuadro 1. Nombre común, origen y acrónimo de las poblaciones de Capsicum spp. colectadas en los estados de Tabasco y Chiapas, México

\begin{tabular}{lllc}
\hline \multicolumn{1}{c}{ Nombre local } & \multicolumn{1}{c}{ Lugar de colecta } & \multicolumn{1}{c}{ Especie } & Acrónimo \\
\hline Amashito & Vicente Guerrero, Teapa, Tabasco & C.a. var. glabriusculum & AVG \\
Amashito & Ejido Cerro Blanco, Tacotalpa, Tabasco & C.a. var. glabriusculum & ACB \\
Pico de Paloma & Ejido Cerro Blanco, Tacotalpa, Tabasco & C. frutescens & PPCB \\
Ojo de Sapo & Ejido Cerro Blanco, Tacotalpa, Tabasco & C.a. & OSCB \\
Amashito Redondo & Miahuatlán, Cárdenas, Tabasco & C.a. var. glabriusculum & ARMI \\
Amashito & Miahuatlán, Cárdenas, Tabasco & C.a. var. glabriusculum & AMI \\
Pico de Paloma & Miahuatlán, Cárdenas, Tabasco & C. frutescens & PPMI \\
Amashito Blanco & Miahuatlán, Cárdenas, Tabasco & C.a. var. glabriusculum & ABMI \\
Amashito & El Macayo, Reforma, Chiapas & C.a. var. glabriusculum & AMA \\
Garbanzo-Pico de Paloma & El Macayo, Reforma, Chiapas & C.a. C. frutescens & GPMA \\
Amashito Gordo & El Macayo, Reforma, Chiapas & C.a. var. glabriusculum & AGMA \\
Pico de Paloma & El Macayo, Reforma, Chiapas & C. frutescens & PPMA \\
Amashito & Reforma, Chiapas & C.a. var. glabriusculum & ARE \\
Pico de Paloma Blanco & Ejido Corralillo, Macuspana, Tabasco & C. frutescens & PPBCR \\
Pico de Paloma & Ejido Corralillo, Macuspana, Tabasco & C. frutescens & PPCR \\
Colmillo de Lagarto & Ejido Corralillo, Macuspana, Tabasco & C.a. & CLCR \\
Amashito & El Porvenir, Macuspana, Tabasco & C.a. var. glabriusculum & APV \\
Corazón de Pollo & Ranchería El Porvenir, Macuspana, Tabasco & C.a. & CPRPV \\
Colmillo de Lagarto & El Porvenir, Macuspana, Tabasco & C.a. & CLPV \\
Corazón de Pollo & El Porvenir, Macuspana, Tabasco & C.a. & CPPV \\
Pico de Paloma & El Porvenir, Macuspana, Tabasco & C. frutescens & PPPV \\
\hline
\end{tabular}

C.a.: Capsicum annuum

Los cuatro marcadores microsatélites o secuencias simples repetidas (SSRs) usados en esta investigación fueron seleccionados de entre los probados por Contreras et al. (2011). Los oligonucleótidos se marcaron con las etiquetas 6FAM y HEX (Applied Biosystems) en el extremo 5' (Cuadro 2) para su detección en un secuenciador de fragmentos por electroforesis capilar. La amplificación en PCR (reacción en cadena de la polimerasa) múltiple se realizó en mezclas de reacción de $25 \mu \mathrm{L}$ que contenía 16,375 $\mu \mathrm{L}$ de $\mathrm{H}_{2} \mathrm{O}$ libre de Nucleasas, $0,5 \mu \mathrm{L}$ iniciador sentido, $0,5 \mu \mathrm{L}$ iniciador antisentido, dNTP's 0,5 $\mu \mathrm{L}$, búfer, PCR nucleótidos $0,5 \mu \mathrm{L}, 5$ XGreen 5 $\mu \mathrm{L}, 0,125 \mu \mathrm{L}$ Taq polimerasa y $2 \mu \mathrm{L}$ de ADN. La amplificación se realizó con una desnaturalización inicial de $4 \mathrm{~min}$ a $94{ }^{\circ} \mathrm{C}, 35$ ciclos de $1 \mathrm{~min}$ a 94 ${ }^{\circ} \mathrm{C}, 1 \mathrm{~min}$ a $65^{\circ} \mathrm{C}, 2 \mathrm{~min}$ a $72^{\circ} \mathrm{C}$ y una extensión final de 12 min a $72{ }^{\circ} \mathrm{C}$. La cantidad y calidad del ADN obtenido se evaluó en geles de agarosa al $1,2 \%$, para ello se utilizó una solución amortiguadora de ácido bórico de sodio $1 \mathrm{X}$ o solución SB como medio conductor para electroforesis de ADN (Brody y Kern, 2004).

Los productos de PCR se corrieron en gel de agarosa al $1,2 \%$ y 160 voltios con intensidad de 50 miliamperes (mA) durante $100 \mathrm{~min}$. Cada gel fue teñido con bromuro de etidio (Sambrook et al., 1989). Los geles se visualizaron en un transiluminador con luz UV. El peso molecular de los fragmentos de ADN obtenidos se visualizó con la ayuda de un marcador de 100-1000 pares de bases ADN Ladder (Promega). La cuantificación de las bandas se realizó con la escala binaria de presencia (1) y ausencia (0).

Análisis estadístico. Las distancias genéticas entre poblaciones se estimaron en base a una matriz de presencia (1) y ausencia (0) de un alelo en un locus. La similitud genética de las poblaciones evaluadas se determinó con el coeficiente de Dice (Nei y Li, 1979), y con ella se generó un dendrograma con el método de agrupamiento de pares no ponderados con medias aritméticas (UPGMA), la altura de corte para 
formar los clusters o grupos se determinó mediante la pseudo estadística $\mathrm{T}$ cuadrada (PS $\mathrm{T}^{2}$ ) y la pseudo $\mathrm{F}$ (Johnson, 2000); el análisis cluster se realizó con el paquete estadístico SAS versión 9.2 (Cary, NC, USA).
Con el propósito de determinar la estructura genética de las poblaciones evaluadas, se realizó un análisis molecular de varianza (AMOVA) y para ello se usó el programa GenAlEx 6,5 (Peakall y Smouse, 2012).

Cuadro 2. Descripción de los cuatro locus usados, motivo que se repite, iniciador y tamaño de cada marcador con los que se realizó la caracterización molecular de las poblaciones de Capsicum spp. de los estados de Tabasco y Chiapas, México

\begin{tabular}{|c|c|c|c|c|}
\hline Locus & Unidad repetitiva & Iniciador & Tamaño (Pb) & $\begin{array}{l}\text { TA } \\
\left({ }^{\circ} \mathrm{C}\right)\end{array}$ \\
\hline HpmsCaSIG19 & (CT)6(AT)8 (GTAT)5 & $\begin{array}{l}\text { D-HEXcatgaatttcgtcttgaaggtccc } \\
\text { R-aagggtgtatcgtacgcagcctta }\end{array}$ & $216-223$ & 54 \\
\hline Hpms1-106 & (AAAAAT)4 & $\begin{array}{l}\text { D-HEXtccaaactacaagcttgcctaacc } \\
\text { R-ttttgcattattgagtcccacagc }\end{array}$ & $158-164$ & 53 \\
\hline Hpms1-143 & (AG)12 & $\begin{array}{l}\text { D-6FAMaatgctgagctggcaaggaaag } \\
\text { R-tgaaggcagtaggtggggagtg }\end{array}$ & $220-232$ & 53 \\
\hline Hpms1-274 & $(\mathrm{GTT}) 7$ & $\begin{array}{l}\text { D-HEX-tcccagacccctcgtgatag } \\
\text { R-tcctgctccttccacaactg }\end{array}$ & $162-180$ & 56 \\
\hline
\end{tabular}

$\mathrm{D}=$ Delante, $\mathrm{R}=$ Reversa, $\mathrm{TA}\left({ }^{\circ} \mathrm{C}\right)=$ Temperatura de alineamiento

\section{RESULTADOS Y DISCUSIÓN}

Los oligonucleótidos HpmsCaSIG19, Hpms1106, Hpms1-143 y Hpms1-274, usados en el presente estudio detectaron en total 229 bandas, con un promedio de 57,25 bandas por oligonucleótido. El promedio de alelos observados en cada población por marcador fue de 2,7. El oligonucleótido HpmsCaSIG19 amplificó 73 bandas, y el marcador que amplificó el menor número de bandas (45) fue Hpms1-274. El número de alelos detectados en las poblaciones de chile evaluadas en la presente investigación representa un resultado similar al reportado por Dhaliwal et al. (2014), Sharmin et al. (2018) y Guzmán et al. (2020). Pero es menor al promedio de alelos encontrados por Nicolaï et al. (2013), quienes con 28 oligonucleótidos microsatélites detectaron 6,64 alelos promedio para los cultivares de $C$. frutescens y 8,8 alelos para las variedades de $C$. annuum evaluadas en su investigación. Inferior número de alelos del presente trabajo también es al promedio de 3,0 alelos por locus que reportan Rivera et al. (2016), a los 4,03 alelos por locus referidos por González et al. (2014), y a los 3,5 alelos por locus que encontraron en su trabajo Hanáček et al. (2009). La posible explicación a esta diferencia pudiera deberse a los marcadores usados en cada investigación, y al material evaluado, ya que en el presente trabajo de las 21 poblaciones evaluadas, 19 de ellas son silvestres y dos criollas, mientras que en las investigaciones referidas se probaron variedades e híbridos.

Los marcadores Hpms1-106, Hpms1-143 y Hpms1-274 probados en el presente estudio también fueron evaluados por Kwon et al. (2005); Contreras et al. (2011), Toledo et al. (2016) y Stavêlíková et al. (2010). Con el oligonucleótido Hpms1-274, Kwon et al. (2005), Hanáček et al. (2009) y Contreras et al. (2011), encontraron un menor número de alelos, mientras que Toledo et al. (2016); y Stavêlíková et al. (2010) con ese mismo marcador, reportaron más alelos en comparación a los hallados en el presente trabajo. Guzmán et al. (2020) usó el marcador HpmsCaSIG19 y encontró que éste detectó 12 alelos en las 42 variedades de chile evaluadas en su estudio. La discrepancia en la cantidad de alelos detectados en los diferentes trabajos de investigación mencionados; no obstante, que se usaron los mismos marcadores, pudiera deberse a que en la presente investigación se caracterizaron poblaciones silvestres y criollas de chile, mientras que en los trabajos de Kwon et al. (2005), Contreras et al. (2011) y Toledo et al. (2016) se evaluaron variedades mejoradas e híbridos de chile.

Del total de las 229 bandas amplificadas por los cuatro marcadores, 70 de ellas fueron 
polimórficas (Cuadro 3), Hpms1-106 fue el iniciador que aportó el mayor porcentaje de polimorfismo debido a que amplificó el 38,88 \% de bandas polimórficas y HpmsCaSIG19 sólo mostró el 20,55 \% de polimorfismo del total de bandas polimórficas. Con respecto al polimorfismo observado, los cuatro marcadores evaluados en la presente investigación detectaron resultados similares a lo reportado por Ulhoa et al. (2014), aunque estos investigadores evaluaron 63 iniciadores en líneas S4 de chile Jalapeño amarillo y sólo el 23,8 \% (15 iniciadores) fueron polimórficos. Del mismo modo, Dhaliwal et al. (2014) de los 50 marcadores evaluados en su investigación, 27 de ellos detectaron polimorfismo.

Cuadro 3. Porcentaje de polimorfismo presentado por las poblaciones silvestres y criollas de Capsicum spp. de los estados de Tabasco y Chiapas, México

\begin{tabular}{lccc}
\hline \multicolumn{4}{c}{ Bandas } \\
\hline \multicolumn{1}{c}{ Locus } & Amplificadas & Polimórficas & Polimorfismo (\%) \\
\hline HpmsCaSIG19 & 73 & 15 & 20,55 \\
Hpms1-106 & 54 & 21 & 38,88 \\
Hpms1-143 & 57 & 18 & 31,58 \\
Hpms1-274 & 45 & 16 & 35,56 \\
\hline Total & 229 & 70 & 30,57 \\
\hline
\end{tabular}

Por otra parte, Patel et al. (2011) indican que tres de los seis marcadores microsatélites o SSRs que usaron en su trabajo identificaron polimorfismo. Huan-huan et al. (2011) reportaron $60,48 \%$ de polimorfismo detectado por los marcadores que se evaluaron en su investigación. Por lo anterior se puede establecer que el polimorfismo que se logre encontrar en Capsicum dependerá en gran medida de los iniciadores usados y los tipos de poblaciones (silvestres, variedades mejoradas, variedades criollas, líneas endocriadas o híbridos) con las que se haya trabajado.

Lo antes señalado se sustenta al comparar los resultados de la presente investigación con lo reportado por Hernández et al. (2006), quien en su trabajo con poblaciones silvestres de chile encontró poco polimorfismo (25 bandas polimórficas de 126 bandas totales detectadas) con RAPDs e Isoenzimas, lo anterior porque en el presente trabajo se encontró 70 bandas polimórficas de 229 bandas observadas. Islam et al. (2016) con tres marcadores TE-AFLP que probaron en 177 accesiones de chile criollo en el noreste de India encontraron $61 \%$ de bandas polimórficas. Bobadilla et al. (2017) con tres RAPDs encontró 30 bandas y de éstas solo nueve fueron polimórficas en variedades de chile. Colney et al. (2018), usando marcadores microsatélites concluyeron que éstos (SSRs) fueron eficientes para detectar alto porcentaje de polimorfismo en los diferentes cultivares de chile evaluados en su trabajo, lo que coincide con los resultados encontrados en la presente investigación.

Los resultados del AMOVA se muestran en el Cuadro 4 y se observa que la varianza entre poblaciones fue de $13,0 \%$, lo que representa un valor bajo si se compara con los valores de 15,75 y 50,0 \% reportados por Pacheco et al. (2012) y Hernández et al. (2006) en poblaciones silvestres, criollas, domesticadas e híbridos de chile del noroeste de México. Y menor también al 92,0 \% reportado por López et al. (2019) en chiles de la Península de Yucatán. La posible explicación a estas discrepancias, podría ser el origen tan diverso de las poblaciones, y al manejo y uso que se le da al germoplasma de chile en cada región.

El valor de la varianza dentro de individuos fue de $87,0 \%$, que supera al 54,0 \% reportado por Islam et al. (2016), al 74,18 \% encontrado por Pacheco et al. (2012), y similar al 86,87 \% de Shiferaw et al. (2018). La varianza estimada entre individuos dentro de las poblaciones (VarEst) mostró un valor de -0,361 que se considera como 0 , por ello el \%VarEst también será 0 (Cuadro 4).

De los tres estadísticos con los que se determinó la estructura genética de las poblaciones evaluadas en esta investigación 
(Cuadro 4), el $\mathrm{F}_{\mathrm{ST}}$ (diferenciación genética entre poblaciones) estimado presentó un valor de $0,176(P \leq 0,001)$, mientras que el $F_{\text {Is }}$ (coeficiente de coancestría) y el $\mathrm{F}_{\mathrm{IT}}$ (coeficiente de endogamia) dieron valores de $-0,448(P=1,000)$ y $-0,193(P=0,999)$, respectivamente.

Cuadro 4. Análisis molecular de varianza de las poblaciones de C. annuum y C. frutescens colectadas en los estados de Tabasco y Chiapas, México, y evaluadas con cuatro marcadores moleculares microsatélites o secuencias simples repetidas (SSRs)

\begin{tabular}{lrrrcc}
\hline \multicolumn{1}{c}{ FV } & GL & SC & CM & VarEst & \% VarEst \\
\hline Entre poblaciones & 20 & 29,540 & 1,477 & 0,172 & 13 \\
Entre individuos dentro de las poblaciones & 42 & 18,667 & 0,444 & 0,000 & 0 \\
Dentro de los individuos & 63 & 73,500 & 1,167 & 1,167 & 87 \\
\hline Total & 125 & 121,706 & & 1,339 & 100 \\
\hline
\end{tabular}

FV =Fuente de variación, Gl= Grados de libertad, $\mathrm{SC}=$ Suma de cuadrados, $\mathrm{CM}=$ Cuadrados medios, VarEst= Varianza estimada, \%VarEst $=$ Porciento de varianza estimada, $\mathrm{F}_{\mathrm{ST}}=0,176^{*}, \mathrm{~F}_{\mathrm{IS}}=-0,448 \mathrm{NS}, \mathrm{F}_{\mathrm{IT}}=-0,193 \mathrm{NS}$

El valor de diferenciación genética entre poblaciones $\left(\mathrm{F}_{\mathrm{ST}}\right)$ encontrado en esta investigación de 0,176 se debe considerar como alto e interpretarse de que las poblaciones evaluadas mostraron mayor diferenciación en función de las frecuencias génicas de cada una de ellas. Se han reportado valores de $\mathrm{F}_{\text {ST }}$ iguales a 0,131 (Shiferaw et al., 2018), 0,108 (Contreras et al., 2011), 0,079 (Toledo et al., 2016) y 0,036 (Hernández et al., 2001), los cuales corresponden a moderada y baja magnitud de diferenciación de las poblaciones. La diferencia en la proporción de cada valor de $F_{S T}$ puede deberse a la naturaleza de las poblaciones de ambos trabajos, en la presente investigación fueron probadas poblaciones de chile silvestre y criollo, en tanto que en los trabajos de los investigadores mencionados se usaron cultivares mejorados. Pacheco et al. (2012) reportó un valor de $\mathrm{F}_{\mathrm{ST}}$ de 0,297 para poblaciones silvestres de chile del Noreste de México, el cual es un valor que se debe considerar como alto con respecto al 0,176 encontrado en esta investigación. El valor de $\mathrm{F}_{\mathrm{IS}}=$ $-0,448$, hace referencia a que las poblaciones evaluadas poseen alto número de heterocigotos dentro de cada una de ellas, mientras que el valor del $F_{\text {IT }}=-0,193$, de que hay poca diferenciación (menor endogamia) en las poblaciones evaluadas, similares resultados a los de esta investigación los reportó Toledo et al. (2016).

El dendrograma (Figura 1) que muestra las relaciones genéticas entre las poblaciones evaluadas en esta investigación está determinado por seis clusters y se determinó con los siguientes parámetros: distancia de 0,95 la pseudoestadística T cuadrada (PST ${ }^{2}$ ) fue 3,6 y la pseudo F (PSF) de
3.1. El cluster I (CI) se formó con 13 poblaciones, entre ellas cinco son Amashitos (AVG Amashito Vicente Guerrero, AGMA Amashito Gordo El Macayo, ACB Amashito Cerro Blanco, AMA Amashito El Macayo y Amashito El Porvenir), Cuatro Pico de Paloma (PPMA Pico de Paloma El Macayo, PPPV Pico de Paloma El Porvenir, PPBCR Pico de Paloma Blanco Corralillo y PPCR Pico de Paloma Corralillo), los dos Colmillo de Lagarto (CLPV Colmillo de Lagarto El Porvenir y CLCR Colmillo de Lagarto Corralillo), las otras dos poblaciones agrupadas en este cluster fueron GPMA (Garbanzo-Pico de Paloma El Macayo) y CPPV (Corazón de Pollo el Porvenir).

Los cluster II, IV y $\mathrm{V}$ se formaron de dos poblaciones cada uno y los cluster III y VI de una población cada uno. La agrupación que se dio en las poblaciones evaluadas en este estudio difiere de lo reportado por González et al. (2011) con poblaciones de Chilpetín quienes encontraron en su trabajo que las poblaciones silvestres se separaron de las criollas, lo que resulta diferente al de la presente investigación, lo cual pudiera deberse a que en algunas localidades las poblaciones se encontraron creciendo y desarrollándose relativamente cerca unas de la otra, por lo que pudo haber ocurrido algún cruzamiento entre las plantas de cada población ya que, aunque Capsicum es considerada como una planta autógama, se ha reportado que puede ocurrir hasta un $30 \%$ de alogamia. De esta forma, alguna población colectada, es decir la que fue objeto del estudio de ADN, pudo realmente corresponder a la población F1. 


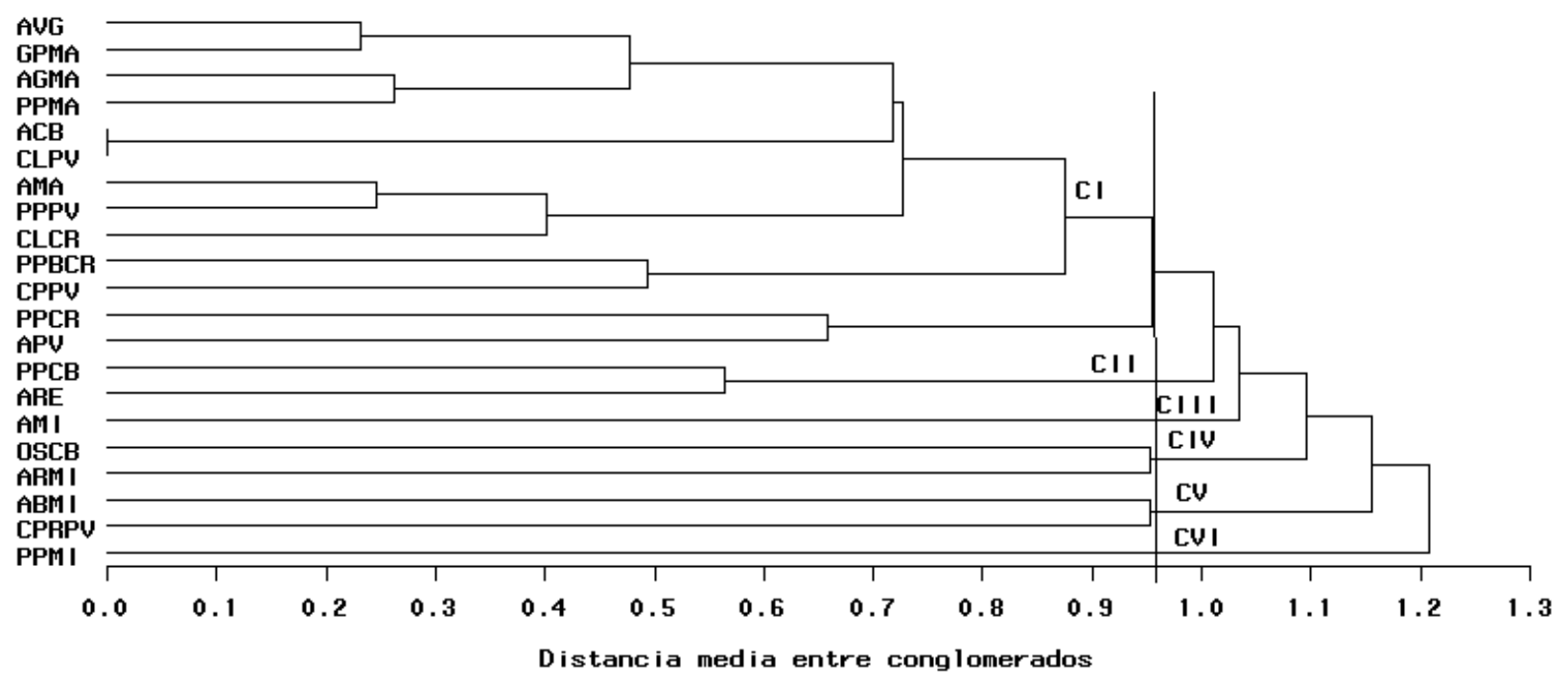

Figura 1. Dendrograma de 21 poblaciones de Capsicum spp. realizado con la distancia de Dice y el método de agrupamiento de pares no ponderados con medias aritméticas (UPGMA).

\section{CONCLUSIONES}

Los cuatro marcadores moleculares evaluados detectaron un porcentaje de alelos polimórficos aceptable en las poblaciones evaluadas y fueron eficientes para identificar su diversidad genética. De la variabilidad estimada por el análisis molecular de varianza el 13,0 \% correspondió a entre poblaciones y $87 \%$ dentro de individuos. Los estadísticos de Wright ( $F_{\text {ST }}, F_{I S}$ y $F_{\text {IT }}$ ) presentaron valores de 0,176, -0,448 y -0,193, y en base a ello se concluye que las poblaciones evaluadas presentan efecto grande de apareamiento no aleatorio de los individuos de cada una de ellas, cuyas posibles causas fueron discutidas con anterioridad. El cluster I, que agrupa a 13 poblaciones, muestra que Amashito Cerro Blanco y Colmillo de Lagarto El Porvenir presentan la menor distancia genética $\mathrm{y}$, por lo tanto, son las poblaciones más parecidas genéticamente. En el cluster VI la población PPMI (Pico de Paloma Miahuatlán) fue la más diferente de todas las poblaciones evaluadas en este estudio.

\section{LITERATURA CITADA}

1. Baba, V.Y., K.R. Rocha, G.P. Gomes, C. de F. Ruas, P. M. Ruas, R. Rodrigues R y L.S. Gonçalves. 2015. Genetic diversity of
Capsicum chinense accessions based on fruit morphological characterization and AFLP markers. Genet Resour Crop Evol 1-10. DOI 10.1007/s10722-015-0325-4

2. Baltazar, B. 1997. Diversidad genética del cultivo de chile (Capsicum spp.) determinada por isoenzimas y RFLPs tipos: serrano, jalapeño, manzano y silvestres en su área de distribución. CONABIO proyecto No. G026. http://www.conabio.gob.mx/institucion/cgi-bin $/$ datos.cgi?Letras $=$ G\&Numero $=26$ (consulta de mayo 16, 2019).

3. Bobadilla-Larios, V., E. Esparza-Ibarra, L. Delgadillo-Ruiz, P. Gallegos-Flores y J. L. Ayala-Lujan. 2017. Variedades de chile (Capsicum annuum L.) identificadas mediante marcadores RAPD. Tropical and Subtropical Agroecosystems 20: 465-473.

4. Brody, J.R. y S.E. Kern. 2004. Sodium boric acid: a Tris-free, cooler conductive medium for DNA electrophoresis. Biotechniques 36(2): 214-216.

5. Carvalho, S.I., L.B. Bianchetti, C.F. Ragassi, C.S. Ribeiro, F.J. Reifschneider, G.S. Buso y F.G. Faleiro. 2017. Genetic variability of a Brazilian Capsicum frutescens germplasm collection using morphological characteristics and SSR markers. Genetics Molecular Research 16(3): 1-18. 
6. Castellón-Martínez, E., J.L. Chávez-Servia, J.C. Carrillo-Rodríguez y A.M. Vera-Guzmán. 2012. Preferencias de consumo de chiles (Capsicum annuum L.) nativos en los valles centrales de Oaxaca, México. Revista Fitotecnia Mexicana 35(5): 27-35.

7. Chamikara, M.D., R.V. Lelwala, M. Ishan, S.S. Karunadasa, M.K. Perera, P.I. Rajapaksha, V.D.W. Kasthuriarachchi, D.T. Jeyakumar, C.K. Weebadde y S.D. Sooriyapathirana. 2015. Morphological and microsatellite marker analysis of fruit size and shape in selected accessions and commercial cultivars of Capsicum species in Sri Lanka. International Journal of Multidisciplinary Studies (IJMS) 2(1): 29-50.

8. Colney, L., W. Tyagi y M. Rai. 2018. Morphological and molecular characterization of two distinct chilli cultivars from North Eastern India with special reference to pungency related genes. Scientia Horticulturae 240: 1-10.

9. Contreras-Toledo, A.R., H. López-Sánchez, A. Santacruz-Varela, E. Valadez-Moctezuma, V.H. Aguilar-Rincón, T. Corona-Torres y P. Antonio-López. 2011. Diversidad genética en México de variedades nativas de chile 'poblano' mediante microsatélites. Revista Fitotecnia Mexicana 34(4): 225-232.

10.Dellaporta, S.L., J. Wood y J.B. Hicks. 1983. Una minipreparación de ADN vegetal: versión II. Reporte de Biología Molecular de Plantas 1(4): 19-21.

11.De Vicente, M.C. y T. Fulton. 2003. Using molecular marker technology in studies on plant genetic diversity. https://www.bioversity international.org/fileadmin/user_upload/online _library/publications/pdfs/Molecular_Markers _Volume_1_en.pdf. (consulta de enero 12, 2020).

12.Dhaliwal, M.S., A. Yadav y S.K. Jindal. 2014. Molecular characterization and diversity analysis in chilli pepper using simple sequence repeats (SSR) markers. African Journal of Biotechnology 13(31): 3137-3143.

13.González, I., Y. Arias, M. Quiñones, I. Miranda, Y. Rodríguez y B. Peteira. 2011. Variabilidad molecular de genotipos de pimiento (Capsicum annuum L.) del programa de mejoramiento genético para la resistencia a PVY. Revista de Protección Vegetal 26(2): 6973.

14.González-Jara, P., A. Moreno-Letelier, A. Fraile, D. Piñero y F. García-Arenal. 2011. Impact of Human Management on the Genetic Variation of Wild Pepper, Capsicum annuum var. glabriusculum. PLoS One 6(12): e28715.

15.González-Pérez, S., A. Garcés-Claver, C. Mallor, L.E. Sáenz de Miera, O. Fayos, F. Pomar, F. Merino y C. Silvar. 2014. New insights into Capsicum spp. relatedness and the diversification process of Capsicum annuum in Spain. PloS One 9(12): e116276.

16.Guzmán, F. A., M.C. De Vicente, S. Moore y M.M. Jahn. 2020. Microsatellites to enhance characterization, conservation and breeding value of Capsicum germplasm. Gen. Res. and Crop Evol. 67(3): 569-585.

17.Hanáček, P., T. Vyhnánek, M. Rohrer, J. Cieslarová y H. Stavělíková. 2009. DNA polymorphism in genetic resources of red pepper using microsatellite markers. Hort. Sci. (Prague) 36(4): 127-132.

18.Haralayya, B. y I.S. Asha. 2017. Molecular Marker Application in Capsicum spp: A Supplement to Conventional Plant Breeding. Int. J. Curr. Microbiol. App. Sci. 6(11): 38403855.

19.Hernández-Verdugo, S., A. GonzálezRodríguez, P. Sánchez-Peña, A. Casas y K. Oyama. 2006. Estructura y diferenciación genética de poblaciones silvestres $\mathrm{y}$ domesticadas de chile del Noroeste de México analizada con isoenzimas y RAPDs. Revista Fitotecnia Mexicana 29(Es2): 25-29.

20.Hernández-Verdugo, S., R. Luna-Reyes y K. Oyama. 2001. Genetic structure and differentiation of wild and domesticated populations of Capsicum annuum (Solanaceae) from Mexico. Plant Systematics and Evolution 226(3-4): 129-142.

21.Huan-huan, H., Z. Zhang-hua, Z. Zheng-hai, M. Sheng-li, W. Li-hao y Z. Bao-xi. 2011. Analysis of SSRs information in Capsicum spp. from EST database. Agricultural Sciences in China 10(10): 1532-1536.

22.Islam, M.A., P. Sinha, S.S. Sharma, M.S. Negi, B. Neog y S.B. Tripathi. 2016. Analysis of 
genetic diversity and population structure in Capsicum landraces from North Eastern India using TE-AFLP markers. Plant Molecular Biology Reporter 34(4): 869-875.

23.Johnson, D. E. 2000. Métodos multivariados aplicados al análisis de datos (No. 519.5 J6). Editorial Paraninfo. 566 p.

24.Konishi, A., N. Furutani, Y. Minamiyama y A. Ohyama. 2019. Detection of quantitative trait loci for capsanthin content in pepper (Capsicum annuum L.) at different fruit ripening stages. Breeding Science 69(1): 30-39.

25.Kwon, Y.S., J.Y. Moon, S.I. Yi, K.M. Bae, E.H. Soh, I.H. Cho y B.D. Kim. 2007. Comparative analysis of pepper (Capsicum annuum L.) varieties using morphological characters, AFLP and SSR markers. Korean Journal of Genetics 29(1): 11-20.

26.Kwon, Y.S, J.M. Lee, G.B. Yi, S.I. Yi, K.M. Kim, E.H. Soh, K.M. Bae, E.K. Park, E.K. Song y B.D. Kim. 2005. Use of SSR markers to complement tests of distinctiveness, uniformity and stability (DUS) of pepper (Capsicum annuum L.) varieties. Molecules \& Cells 19(3): 428-435.

27.Latournerie, L., J.L. Chávez, M. Pérez, G. Castañón, S.A. Rodríguez, L.M. Arias y P. Ramírez. 2002. Valoración in situ de la diversidad morfológica de chiles (Capsicum annuum L. y Capsicum chinense Jacq.) en Yaxcabá, Yucatán. Revista Fitotecnia Mexicana 25(1): 25-33.

28.López Castilla, L.D., R. Garruña Hernández, C.D. Castillo Aguilar, A. Martínez-Hernández, M.M. Ortiz-García y R.H. Andueza-Noh. 2019. Structure and genetic diversity of nine important landraces of Capsicum species cultivated in the Yucatan Peninsula, Mexico. Agronomy 9(7): 376.

29.MacNeish, R.S. 1964. Ancient mesoamerican civilization. Science 143(3606): 531-537.

30.Martínez-Sánchez, D., M. Pérez-Grajales, J.E. Rodríguez-Pérez y E. del C. Moreno-Pérez. 2010. Colecta y caracterización morfológica de 'chile de agua' (Capsicum annuum L.) en Oaxaca, México. Revista Chapingo. Serie Horticultura 16(3): 169-176.

31.Massot, P.H. y R.L. Barbieri. 2016. Plant breeding of chili peppers (Capsicum,
Solanaceae) - A review. Australian Journal of Basic and Applied Sciences 10(15): 148-154.

32.Nei, M. y W.H. Li. 1979. Mathematical model for studying genetic variation in terms of restriction endonucleases. Proceedings of the National Academy of Sciences 76(10): 52695273.

33.Nicolaï, M., M. Cantet, V. Lefebvre, A.M. Sage-Palloix y A. Palloix. 2013. Genotyping a large collection of pepper (Capsicum spp.) with SSR loci brings new evidence for the wild origin of cultivated $C$. annuum and the structuring of genetic diversity by human selection of cultivar types. Genetic Resources and Crop Evolution 60(8): 2375-2390.

34.Pacheco-Olvera, A., S. Hernández-Verdugo, V. Rocha-Ramírez, A. González-Rodríguez y K. Oyama. 2012. Genetic diversity and structure of pepper (Capsicum annuum L.) from Northwestern Mexico analyzed by microsatellite markers. Crop Science 52(1): 231-241.

35.Patel, A.S., N. Sasidharan y A.G. Vala. 2011. Research article genetic relation in Capcicum annum L. cultivars through microsatellite markers: SSR and ISSR. Electronic Journal of Plant Breeding 2(1): 67-76.

36.Peakall, R. y P.E. Smouse. 2012. GenAlEx 6,5: genetic analysis in Excel. Population genetic software for teaching and research - an update. Bioinformatics 28: 2537-2539.

37.Rahevar, P.M., J.N. Patel, S. Kumar y R. Acharya. 2019. Morphological, biochemical and molecular characterization for genetic variability analysis of Capsicum annuum. Vegetos 32(2): 131-141.

38. Rivera, A., A. Monteagudo, E. Igartuac, A. Taboada, A. García-Ulloa, F. Pomar, M. Riveiro-Leira y C. Silvar. 2016. Assessing genetic and phenotypic diversity in pepper (Capsicum annuum L.) landraces from NorthWest Spain. Scientia Horticulturae 203: 1-11.

39.Sambrook, J., E. Fritsch y T. Maniatis. 1989. Molecular Cloning: A Laboratory Manual. Cold Spring Harbor Laboratory Press. New York.

40.Sharmin, A., M.E. Hoque, M.M. Haque y F. Khatun. 2018. Molecular diversity analysis of some chilli (Capsicum spp.) genotypes using 
SSR markers. American Journal of Plant Sciences 9(3): 368-379.

41.Shiferaw, A., A. Destaw y G. Samuel. 2018. Genetic diversity study of Ethiopian hot pepper cultivars (Capsicum spp.) using Inter Simple Sequence Repeat (ISSR) marker. J. Plant Mol. Breed. 5(2): 27-37.

42.Stavêlíková, H., P. Hanácek y T. Vyhnánek. 2010. The morphological description and DNA tools analysis: for detection of duplicitions in the Czech germplasm collection of pepper (Capsicum annuum L.). Acta Univ. Agric. et Silvic. Mendel. Brun. LVIII (1): 191198.

43.Thul, S.T., M.P. Darokar, A.K. Shasany y S.P. Khanuja. 2012. Molecular profiling for genetic variability in Capsicum species based on ISSR and RAPD markers. Molecular Biotechnology 51(2): 137-147.

44.Toledo-Aguilar, R., H. López-Sánchez, A.
Santacruz-Varela, E. Valadez-Moctezuma, P. Antonio-López, V.H. Aguilar-Rincón, V.A. González-Hernández y H. Vaquera-Huerta. 2016. Characterization of genetic diversity of native 'Ancho' chili populations of Mexico using microsatellite markers. Chilean Journal of Agricultural Research 76(1): 18-26.

45.Ulhoa, A.B., T.N. Pereira, R.N. Silva, C.F. Ragassi, R. Rodrigues, M.G. Pereira y F.J. Reifschneider. 2014. Caracterização molecular de linhagens de pimenta do tipo Jalapeño amarelo. Horticultura Brasileira 32(1): 35-40.

46.Xiao-min, Z., Z. Zheng-hai, G. Xiao-zhen, M. Sheng-li, L. Xi-xiang, J. Chadœuf, A. Palloix, W. Li-hao y Z. Bao-xi. 2016. Genetic diversity of pepper (Capsicum spp.) germplasm resources in China reflects selection for cultivar types and spatial distribution. Journal of Integrative Agriculture 15(9): 19912001. 